\title{
The current distribution and status of leopards Panthera pardus in China - CORRIGENDUM
}

Alice LaguardiaA, Jan F. Kamlera, Sheng Lia

ChengCheng Zhanga, Zhefeng Zhoua and Kun Shia

Doi: 10.1017/S0030605315000472. Published online by Cambridge University Press, 30 October 2015

In Fig. 1 of Laguardia et al. (2015) the record for Resuo county (Resuoxian) was positioned incorrectly in Resuo township (Resuoxiang). We thank Joerg Hensiek for pointing this out. The correct figure is provided here.

\section{Reference}

Alice Laguardia, Jan F. Kamler, Sheng Li, Chengcheng Zhang, Zhefeng Zhou and Kun SHI. The current distribution and status of leopards Panthera pardus in China. Published 30 October 2015, doi:10.1017/Soo30605315000472

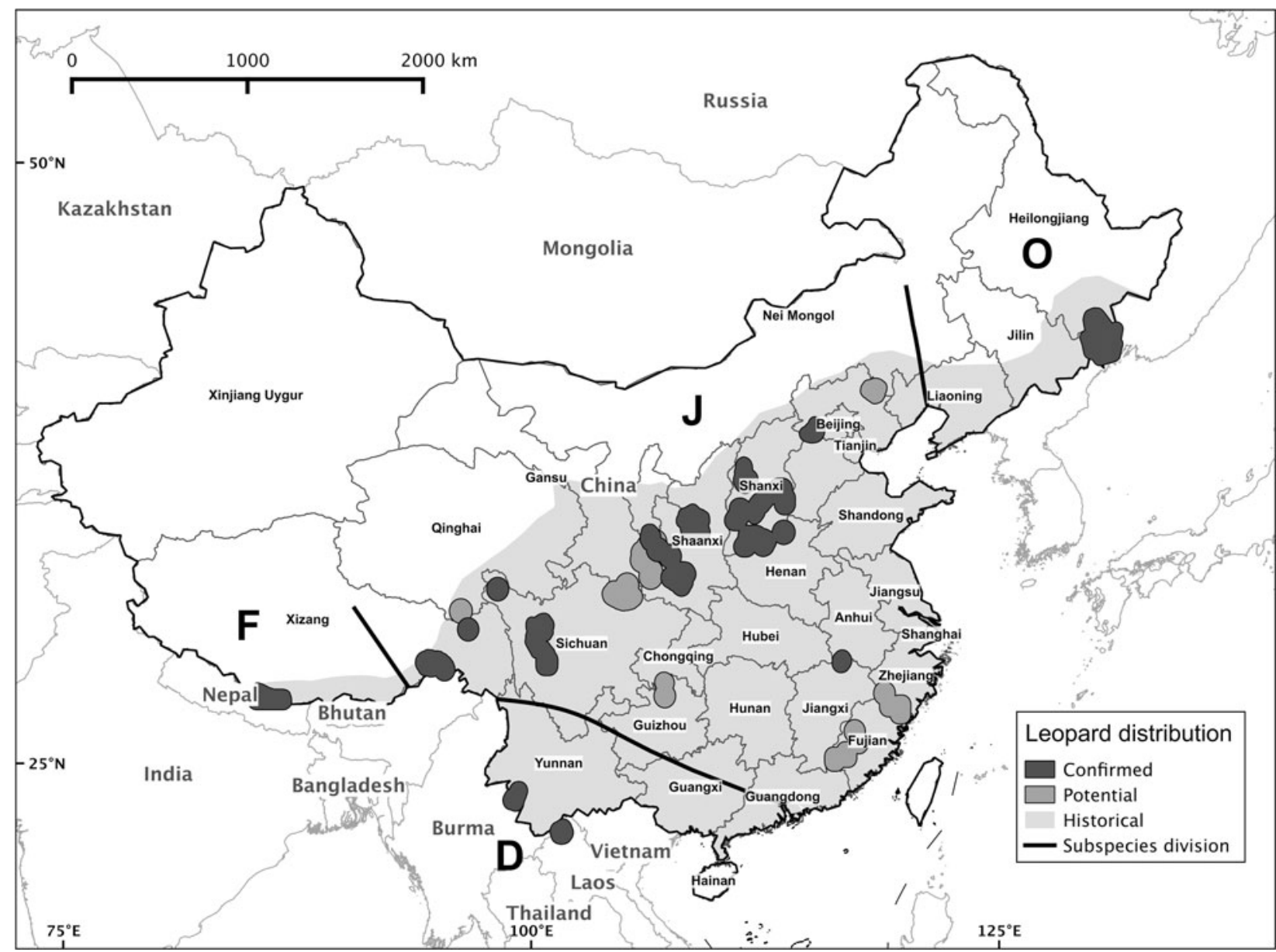

